\title{
La sobrexpresión génica vascular de NADPH oxidasa en la hipertensión arterial experimental se reduce solamente con inhibición directa de Rho kinasa
}

Jorge Jalil, Ulises Novoa, Italo Mora, María Paz Ocaranza

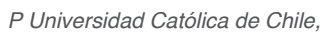

Financiamiento: Fondecyt 1085208

\section{Resumen:}

Uno de los objetivos del tratamiento antihipertensivo, más allá de normalizar las cifras tensionales, es disminuir/regresar el remodelado patológico hipertensivo cardiovascular y renal, de manera de reducir eficientemente el riesgo dado por esta patología. $\mathrm{Al}$ respecto, la vía de señalización intracelular de la Rho kinasa (ROCK) es un mecanismo de vasoconstricción y de promoción de remodelado que podría ser un blanco atractivo en el tratamiento antihipertensivo.

Objetivo: Evaluar a nivel vascular los niveles de TGF beta, la expresión génica vascular de NADPH oxidasa (fuente de stress oxidativo vascular), y el grado de inflamación parietal y su dependencia de ROCK en la hipertensión arterial (HTA) experimental.

Métodos: Se compararon 5 grupos experimentales. Se usó el modelo de HTA por administración de deoxicorticosterona (DOCA, $100 \mathrm{mg} / \mathrm{Kg}$, sc una vez/semana) + sal en ratas Sprague Dawley uninefrectomizadas. Como controles, se usaron ratas uninefrectomizadas (Sham). Las ratas DOCA se randomizaron para recibir el inhibidor específico de ROCK fasudil (Fas, $50 \mathrm{mg} / \mathrm{kg} / \mathrm{d}$, por gavage, durante 3 semanas), desde la semana 3 post cirugía, o candesartán (Cand, $10 \mathrm{mg} / \mathrm{kg} / \mathrm{d}$, por gavage, durante 3 semanas), o fasudil $(25 \mathrm{mg} / \mathrm{kg} / \mathrm{d})+$ candesartan $(5 \mathrm{mg} /$ $\mathrm{kg} / \mathrm{d}$ por gavage, 3 semanas ). Al finalizar los experimentos se midieron en la aorta los niveles de MYPT1 fosforilada/total, blanco de ROCK y estimador de su activación (por Western blot), de TGF beta (por Western blot), los niveles de mRNA de las subunidades p22 Phox y gp 91 de la NADPH oxidasa (por RT PCR) y el número de cé- lulas inflamatorias ED1 en anillos aórticos (por inmunohistoquímica).

Resultados: La presión arterial sistólica aumentó en las ratas DOCA a $172 \pm 7 \mathrm{~mm} \mathrm{Hg}(\mathrm{p}<0,05)$ y fue normalizada después de 3 semanas de tratamiento con candesartán, fasudil y de candesartán + fasudil. La actividad de ROCK en aorta aumentó en 4 veces en las ratas hipertensas $(\mathrm{p}<0,05)$ y se normalizó con los 3 tratamientos antihipertensivos. En las ratas hipertensas la expresión génica en aorta de las subunidades de NADPH oxidasa p22 Phox y gp91 aumentó significativamente $(\mathrm{p}<0.05)$ en 80 y $100 \%$, respectivamente y se redujo significativamente a valores normales en las ratas que recibieron fasudil y candesartán + fasudil. La expresión génica en la aorta de TGF beta aumentó en las ratas hipertensas 4 veces y se normalizó en los 3 grupos tratados. El número de células ED1 en la pared aórtica aumentó 6 veces en las ratas hipertensas y disminuyó significativamente con los 3 tratamientos.

Conclusiones: En este modelo de HTA experimental hay un importante aumento de la actividad de ROCK en la pared aórtica. El tratamiento antihipertensivo previene la inflamación y la sobrexpresión génica de TGF beta en la pared vascular. La sobrexpresión génica de NADPH oxidasa en la pared aórtica en las ratas hipertensas se normaliza solamente al inhibir en forma directa ROCK con un inhibidor específico. Es posible la inhibición directa de ROCK sea una forma más completa de tratamiento antihipertensivo, al prevenir la expresión de las subunidades de NADPH oxidasa p22 Phox y gp91 en la pared aórtica, hecho que no se observó utilizando un antihipertensivo de última generación. 


\section{Vascular gene overexpression of NADPH oxydase in experimental hypertension is only reduced by direct Rho kinase inhibition}

Background: Rho kinase (ROCK) activity promotes vasoconstriction and pathological vascular remodeling in experimental hypertension. Our working hypothesis is that ROCK inhibition could be an attractive target to prevent vascular remodeling in hypertension.

Objectives: We evaluated vascular TGF beta, the genic expression of NADPH oxydase (a vascular oxidative stress source) and its dependency from ROCK activation in experimental hypertension in the rat.

Methods: Five experimental groups were compared. Hypertension was induced by the administration of salt and deoxycorticosterone acetate (DOCA, $100 \mathrm{mg} / \mathrm{Kg}$, weekly) to unilaterally nephrectomized rats. Unilaterally nephrectomized rats were used as controls (controls). DOCA rats were randomized to receive either Fasudil (a ROCK inhibitor, $50 \mathrm{mg} / \mathrm{Kg} /$ day) or candesartan (CAND, $10 \mathrm{mg} / \mathrm{Kg} / \mathrm{day}$ for 3 weeks), starting 3 weeks after surgery. The other group received fasudil $(25 \mathrm{mg} / \mathrm{Kg} / \mathrm{day})$ plus CAND ( $5 \mathrm{mg} / \mathrm{Kg} /$ day) for 3 weeks. After treatment, phosphorilated MYPT1 (a ROCK target expressing ROCK activation) was measured in aortic wall rings by Western Blot. We also determined TGF-beta (Western

\section{Introducción:}

Un problema clínico-epidemiológico de alta prevalencia es el alto riesgo cardiovascular (ARCV) en pacientes con hipertensión arterial (HTA) a pesar del tratamiento antihipertensivo actual. En los pacientes hipertensos, el ARCV se asocia a mayor morbimortalidad por accidentes cerebrovasculares, síndromes coronarios agudos e insuficiencia cardíaca. El ARCV está dado por la presencia de factores de riesgo cardiovascular (tres o más), compromiso de órganos blancos, de diabetes mellitus o de otras condiciones clínicas asociadas (enfermedad cerebrovascular, cardiopatía, nefropatía, enfermedad vascular periférica o retinopatía avanzada) ${ }^{1,2}$. En Chile la prevalencia estimada de ARCV global es del 54.9\% de la población ${ }^{3}$.

Un desafío actual en el manejo de la HTA es la prevención/regresión del remodelado cardiovascular y renal patológico - factores fundamentales en el aumento del riesgo cardiovascular - por lo cual el conocimiento más preciso de los mecanismos causantes de remodelado cardiovascular y renal llevará a su prevención/regresión
Blot), p22 Phox and gp 91subnits of NADPH oxydase mRNA (RT-PCR) and the number of ED1 inflammatory cells.

Results: In DOCA rats, SBP increased to $172 \pm 7 \mathrm{~mm}$ $\mathrm{Hg}(\mathrm{p}<0,05)$, and returned to normal values after 3 weeks with candesartan, fasudil or both combined. In these rats, ROCK activity in aorta was increased 4 times ( $\mathrm{p}<$ $0,05)$ and returned to control values in the 3 groups receiving treatment. p22 Phox and gp 91subnits of NADPH oxydase mRNA were increased by 80 and $90 \%$, respectively $(\mathrm{p}<0,05)$. These changes were reduced to control values in rats receiving fasudil and candesartan + fasudil. Gene expression of TGF-beta increased 4 times, and the number of ED1 cells increased 6 times in the DOCA rats and returned to normal values in the groups treated with fasudil or candesartan + fasudil.

Conclusion: Antihypertensive treatment was able to prevent inflammation and gene over expression of TGF beta at the vascular wall level. NADPH oxydase overexpression was normalized only after the direct inhibition of ROCK by a specific ROCK inhibitor.

Keywords: vascular NADPH oxydase, Rho kinase

y en consecuencia a disminuir el ARCV y renal en estos pacientes. La vía de señalización intracelular de la RhoA / Rho-kinasa (ROCK) es un mecanismo novedoso, con una participación significativa en el remodelado patológico, descubierto en la última década. Esta vía es activada por agonistas de receptores acoplados a la proteína G de membrana, como angiotensina II (Ang II), endotelina o noradrenalina, y produce contracción de las células musculares lisas e HTA. Además, la vía RhoA-ROCK participa en procesos celulares que intervienen en la patogenia de diversas enfermedades cardiovasculares y renales, ya que participa en los efectos de moléculas vasoactivas y promotoras de remodelado cardiovascular y renal, como angiotensina II, 5-hidroxitriptamina, trombina, factor de crecimiento derivado de plaquetas, endotelina, noradrenalina, tromboxano A2 y urotensina II ${ }^{4-6}$.

La activación de ROCK, blanco de Rho A, produce además una cadena de eventos celulares como de regulación de la óxido nítrico sintasa endotelial por disminución de su expresión génica, activación de NADPH oxidasa con aumento del estrés oxidativo, lo que lleva a 
aumento de TGF, PKC y de MAPK-ERK $1 \frac{1}{2}{ }^{7,8}$.

Así, ROCK promueve stress oxidativo y remodelado.

Sin embargo, el rol de la presión arterial v/s la activación de ROCK en la sobrexpresión vascular de NADPH oxidasa en la HTA, no ha sido evaluado.

El objetivo del presente estudio ha sido evaluar la expresión génica vascular de NADPH oxidasa - fuente de stress oxidativo vascular -, su relación con parámetros de remodelado vascular y, fundamentalmente, su dependencia de Rho kinasa (ROCK) en la hipertensión arterial (HTA) experimental.

\section{Métodos:}

El estudio fue realizado en el Laboratorio de Cardiología Molecular del Departamento de Enfermedades de la Pontificia Universidad Católica (PUC) de acuerdo a la Guía para el cuidado y uso de animales de laboratorio, publicado por el National Health Institute (NIH N ${ }^{\circ} 85$ $23,1985)$ y fue previamente aprobado por la Comisión de Investigación de la Pontificia Universidad Católica de Chile.

Modelo experimental: Se utilizó el modelo de HTA experimental por sobrecarga de volumen mediante administración del mineralocorticoide deoxicorticosterona (DOCA) y sal, en ratas Sprague Dawley machos de $(150 \pm 10 \mathrm{~g})$. En este modelo, bajo anestesia intraperitoneal con xilaxina y ketamina, se realizó nefrectomía izquierda. Posteriormente se administró DOCA (100 $\mathrm{mg} / \mathrm{Kg}$ de peso, Steraloids. Inc., Newport, RI, USA) una vez por semana por vía intraperitoneal, partiendo inmediatamente después de efectuada la cirugía durante seis semanas. Los animales recibieron, además, el agua de bebida con $1 \%$ de $\mathrm{NaCl}$ y $0,4 \% \mathrm{KCl}$.

Después de tres semanas de administración exclusiva de DOCA, las ratas hipertensas DOCA-sal fueron divididas en cuatro grupos en forma randomizada:

- Ratas que recibieron suero fisiológico por gavage + DOCA ip semanal durante tres semanas adicionales, (grupo DOCA),

- Ratas que recibieron el antagonista del receptor de angiotensina II, candesartán (10 mg/kg/día, Laboratorios Saval, Santiago de Chile por gavage + DOCA ip semanal durante tres semanas adicionales (grupo DOCA-cand).

- Ratas que recibieron el inhibidor de Rho kinasa fasudil $(50 \mathrm{mg} / \mathrm{Kg} / \mathrm{dia}$, LC Laboratories, Woburn, MA, USA.) por gavage + DOCA ip semanal durante tres semanas adicionales (grupo DOCA-fas).
- Ratas que recibieron la combinación de ambos tratamientos; candesartán y fasudil (5 y $25 \mathrm{mg} / \mathrm{Kg}$ /dia respectivamente) por gavage + DOCA ip semanal durante tres semanas adicionales, (grupo DOCA-cand-fas).

Un quinto grupo de ratas se utilizó como control. Este grupo fue sometido a nefrectomía izquierda y recibió suero fisiológico vía gavage durante seis semanas en total (grupo SHAM).

Todos los animales fueron mantenidos bajo condiciones controladas de luz y oscuridad y tuvieron libre acceso a agua y comida. Al término de las seis semanas de tratamiento total, los animales fueron sacrificados bajo anestesia con Ketamina (35mg/Kg peso) y Xilazina ( $7 \mathrm{mg} / \mathrm{Kg}$ peso) vía ip. La aorta se extrajo rápidamente, se lavó en suero fisiológico, se cortó en secciones de $15 \mathrm{~mm}$ de longitud y se congeló en nitrógeno líquido, guardándola a $-80^{\circ} \mathrm{C}$ hasta su procesamiento. La presión arterial sistólica (PAS) se midió semanalmente por pletismografía en la cola de los animales bajo suave anestesia con éter etílico y el grado de hipertrofia ventricular izquierda se estimó como la masa ventricular izquierda relativa $(\mathrm{MVIR}=\mathrm{VI} * 100 /$ peso $)$.

Preparación de los extractos de aorta para Western blot: La aorta fue homogenizada en buffer de lisis frío (Tris $\mathrm{HCl} 50 \mathrm{mmol} / \mathrm{L}, \mathrm{NP} 40$ 1\%, NaCl $150 \mathrm{mmol} / \mathrm{L}$, Na-deoxicolato $0.25 \%$, EDTA $1 \mathrm{mmol} / \mathrm{L}$, SDS $0.1 \%$, aprotinina $1 \mu \mathrm{g} / \mathrm{mL}$, leupeptina $1 \mu \mathrm{g} / \mathrm{mL}$ and PMSF 1 $\mathrm{mmol} / \mathrm{L})$. Las muestras fueron centrifugadas a $4^{\circ} \mathrm{C}$ y el contenido de proteínas del supernadante se determinó por Bradford utilizando albúmina de suero de bovino como standard. Las fracciones solubles se calentaron a $95^{\circ} \mathrm{C}$ con 0.33 vol. de 4 X SDS-PAGE buffer para Western blot (PAU).

Fosforilación de la proteína blanco 1 de la fosfatasa de la miosina (myosin phosphatase target protein-1 o MYPT1), MYPT1 en aorta como estimación de la actividad enzimática de Rho kinasa (Western blot) $\left({ }^{9)}\right.$ : Se homogenizó el tejido en $500 \mu \mathrm{L}$ de tampón de lisis, se centrifugó a 10.000 g por 30 minutos a $4^{\circ} \mathrm{C}$, separando el sobrenadante. Para la determinación de los niveles de MYPT1 por western blot (WB), se separaron 100 $\mu \mathrm{g}$ del extracto proteico por electroforesis en geles SDS-PAGE al 6\%. Luego las proteínas se transfirieron a una membrana de nitrocelulosa a $300 \mathrm{~mA}$ durante 1 h. La membrana posteriormente se bloqueó en solución PBS+Tween 20 al 0,05\% con leche descremada al 7\% por $2 \mathrm{~h}$ en agitación constante a temperatura ambiente. Se hicieron 3 lavados con PBS+Tween, conteniendo 
0,5\% de leche descremada (esta solución se utilizó para todos los lavados posteriores y para la dilución de los anticuerpos), la membrana se incubó con el anticuerpo anti-MYPT1 fosforilado (Thr 696, Millipore) con una dilución de 1:2000 y el anticuerpo anti-MYPT1 total (BD Transduction Laboratories) con una dilución de 1:1000 se incubaron toda la noche con agitación a $4^{\circ} \mathrm{C}$. Posteriormente se continuó con 3 lavados similares a los anteriormente descritos y luego se incubó con el anticuerpo secundario unido a peroxidasa (anti-IgG de conejo, para MYPT1 fosforilada y anti-IgG de ratón, para MYPT1 total, PIERCE) en una dilución 1:10000 y 1:5000 respectivamente durante $2 \mathrm{hrs}$. a temperatura ambiente con agitación. Se hicieron 3 lavados por 10 minutos c/u y la membrana se incubó con el sustrato de quimioluminiscencia según las instrucciones del proveedor (Western Lightning ${ }^{\text {TM }}$ Plus-ECL, Perkin Elmer). La intensidad de cada banda se analizó por densitometría mediante un scanner Hewlett-Packard y el software Image J $1.32 \mathrm{j}$ (NIH, USA). Los resultados se expresan como razón MYPT1 fosforilada / MYPT1 total.

\section{Niveles de TG $\beta 1$ en tejido aórtico (Western blot):}

Los niveles proteicos de TG $\beta 1$ en la aorta se determinaron por Western blot. En los extractos de aorta se determinó la concentración de proteínas, las que se cargaron y separaron por SDS-PAGE en geles de poliacrilamida al $12 \%$ y fueron electrotransferidas a nitrocelulosa. Estas membranas de nitrocelulosa fueron bloqueadas con leche baja en grasas (al 5\%) en PBS y colocadas en Tween- 20 a $4^{\circ} \mathrm{C}$ durante una noche. El anticuerpo primario anti-TGF 1 -1 (Santa Cruz, California) se diluyó en leche baja en grasas (0.5\% en PBS conteniendo $0.005 \%$ Tween-20, PBST, 1:1000). Las membranas de nitrocelulosa se incubaron con el anticuerpo primario durante $6 \mathrm{hrs}$. a temperatura ambiente. Luego de lavar con PBST, los blots se incubaron con el anticuerpo secundario unido a horseradish peroxidase $(1 / 10,000)$ durante $2 \mathrm{hrs}$. Los blots fueron lavados nuevamente en PBST y la unión específica se detectó usando ECL con exposición a un film Kodak. La intensidad de cada banda se analizó por densitometría mediante un scanner y el software Image J $1.32 \mathrm{j}(\mathrm{NIH}, \mathrm{USA})^{9}$

Expresión génica en aorta de las subunidades $p 22$ Phox y GP-91phox de NADPH oxidasa por RT-PCR. Brevemente, el RNA total de la aorta se aisló por el método del trizol y se cuantificó por espectroscopía a $260 \mathrm{~nm} / 280 \mathrm{~nm}$. El cDNA se obtuvo por transcripción reversa a partir de $1,5 \mu \mathrm{g}$ de RNA total tratado con DNAsa. La amplifica- ción para GP-91phox (40 ciclos) fue realizada con $1 \mathrm{~min}$ a $94^{\circ} \mathrm{C}$ de denaturación, $1 \mathrm{~min}$ a $55^{\circ} \mathrm{C}$ de hibridización y 1 min a $72{ }^{\circ} \mathrm{C}$ de elongación, seguido por una autoextensión de $10 \mathrm{~min}$ a $72^{\circ} \mathrm{C}$. Como partidor sentido se usó: $5^{\prime}$ AGC-TAG-AGT-GGC-ACC-CAT-TC $3^{\prime}$ y antisentido: 5'-TCC-CAG-TTG-GGC-CGT-CCA-TA- 3' (26). La amplificación para P22phox (40 ciclos) fue realizada con $1 \mathrm{~min}$ a $94^{\circ} \mathrm{C}$ de denaturación, $1 \mathrm{~min}$ a $55^{\circ} \mathrm{C}$ de hibridización y $1 \mathrm{~min}$ a $72^{\circ} \mathrm{C}$ de elongación, seguido por una autoextensión de $10 \mathrm{~min}$ a $72^{\circ} \mathrm{C}$. Como partidor sentido se usó: 5'GTT-TGT-GTG-CCT-GCT-GGA-GT-3' y antisentido: 5 'TGG-GCG-GCT-GCT-TGA-TGG-T 3' ${ }^{10}$. La intensidad de las bandas se cuantificó por densitometría y se normalizó con respecto a la banda $18 \mathrm{~S}$ del RNA ribosomal.

Presencia y cuantificación de macrófagos/monocitos (células ED1) en anillos aórticos: Se fijó en solución de Bouin un trozo de aorta por rata. Posteriormente los trozos fueron deshidratados e incluidos en Paraplast ${ }^{\circledR}$ plus (Sigma Chemicals, St. Louis, MO, USA.). Para inmunohistoquímica se utilizaron secciones de $4 \mu \mathrm{m}$ de espesor montadas en portaobjetos. La inmunotinción fue realizada por el método de peroxidasa anti-peroxidasa (PAP) descrito por Sternberger ${ }^{11}$ con modificaciones. Brevemente, los anillos aórticos fijados fueron desparafinados, rehidratados y tratados con $3 \%$ de peróxido de hidrógeno en metanol absoluto (vol/vol) por $15 \mathrm{~min}$ para inhibir la actividad de las pseudo peroxidasas endógenas. Se realizó luego una recuperación antigénica, hirviendo las muestras en horno de microondas con una solución de EDTA 3 veces. Luego los cortes se lavaron con tampón Tris fosfato salino y se incubaron toda la noche a $4^{\circ} \mathrm{C}$ con el anticuerpo monoclonal contra el antígeno ED-1 de macrófagos/monocitos (Mouse anti rat CD68, MCA341R, Serotec) a una dilución de 1:200. La inmunotinción se reveló con el kit LSAB+System-HRP (Dako, UK) y las secciones fueron contrastadas con hematoxilina, deshidratadas y aclaradas con xilol. Se cuantificó el número de células teñidas en cada anillo (3 anillos/rata)

Análisis estadístico: Los datos obtenidos se expresan como promedio $\pm \mathrm{ES}$. Cada grupo experimental estuvo constituido por 12 animales. Para las comparaciones se usó análisis estadístico con ANOVA de un factor seguido de prueba de $t$ de Student- Newman-Keuls. El análisis estadístico se realizó usando el programa estadístico SPSS 10.0. Se consideró $4 \mathrm{n}$ valor de $\mathrm{p}<0,05$ como estadísticamente significativo. 


\begin{tabular}{llllll|}
\multicolumn{5}{c}{ Tabla 1: Presión arterial e hipertrofia ventricular izquierda } \\
\hline & SHAM & DOCA & DOCA-Cand & DOCA-Fas & DOCA-Cand-Fas \\
\hline PAS (mm Hg) & $117 \pm 2^{*}$ & $172 \pm 7$ & $108 \pm 2^{*}$ & $97 \pm 6^{*}$ & $128 \pm 2^{*}$ \\
MVIR ( VI * 100/peso) & $330 \pm 1^{*}$ & $540 \pm 3$ & $450 \pm 2^{*}$ & $470 \pm 2^{*}$ & $390 \pm 2^{*}$ \\
& & & & & \\
\hline
\end{tabular}

Tabla 1. Valores como promedio $\pm E S ; N=12$ / grupo experimental. Abreviaciones: Cand $=$ candesartán; Fas = fasudil $; P A S=$ presión arterial sistólica $; . M V I R=$ masa ventricular izquierda relativa $; V I=$ ventrículo izquierdo. Símbolos: $*=p<0.05$ vs DOCA después de ANOVA significativo.

\section{Resultados:}

Presión arterial e hipertrofia ventricular izquierda:

Las ratas tratadas solamente con DOCA durante 6 semanas se hicieron hipertensas, superando al final del estudio en promedio en $55 \mathrm{~mm} \mathrm{Hg}$ de PA sistólica a las ratas Sham $(\mathrm{p}<0.05)$. Además, las ratas DOCA presentaron hipertrofia ventricular izquierda (HVI) considerable (63\% por sobre las ratas Sham; $\mathrm{p}<0.05$, Tabla 1).

En los tres grupos bajo tratamiento (DOCA + Candesartán, DOCA + Fasudil y DOCA + Fasudil + Candesartán) se observó normotensión, sin diferencias significativas en- tre los grupos y menor grado de HVI (Tabla 1).

Actividad de Rho kinasa:

La actividad de Rho kinasa en la pared aórtica, medida como niveles de fosforilación de la proteína blanco MYPT1 aumentó 4 veces en las ratas DOCA en comparación con las ratas Sham ( $\mathrm{p}<0.05$, Figura 1). Después de tres semanas de tratamiento con Candesartán, Fasudil o con la combinación de ambos, la actividad de Rho kinasa en la pared aórtica fue similar en estos tres grupos respecto de las ratas Sham, sin diferencias entre ellos (Figura 1).

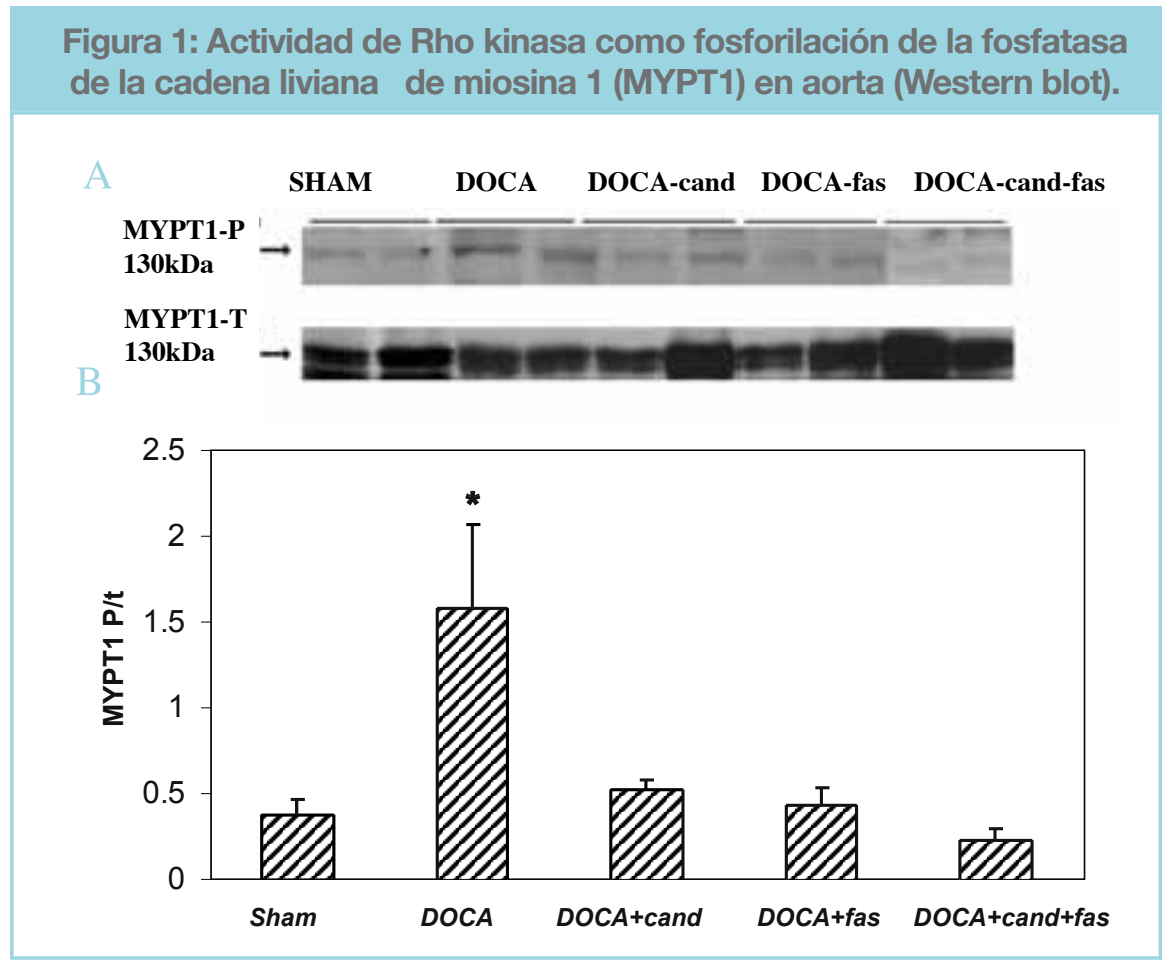

Figura 1.Expresada como relación MYPT1 P/t (fosforilada vs total). En panel superior se observan Western blots representativos en cada grupo. Promedio $\pm E S ; N=8-12$ / grupo. Símbolos: $*=p<$ 0.05 vs todos los otros grupos, post ANOVA significativo 
Expresión génica ( $m R N A)$ de las subunidades p22 Phox y gp 91 de la NADPH oxidasa: La expresión génica (mRNA) de las subunidades p22 Phox y gp 91 de la NADPH oxidasa medidas en la pared aórtica por RT- PCR aumentó en las ratas DOCA en 80 y $100 \%$ respectivamente en comparación con las ratas sham $(\mathrm{p}<0.05$, Figuras 2 y $3)$. Niveles elevados similares a los de las ratas hipertensas
DOCA de ambas subunidades de NADPH en la pared aórtica se observaron en el grupo DOCA + Candesartán. Sin embargo, en ambos grupos tratados con fasudil (grupo DOCA + Fasudil y grupo DOCA + Fasudil + Candesartán) los niveles de mRNA en la pared aórtica de ambas subunidades de NADPH fueron similares a los de las ratas controles normotensas (grupo sham, Figuras 2 y 3 ).

Figura 2: mRNA de la subunidad p22 de la NADPH oxidasa (RT PCR) en aorta.

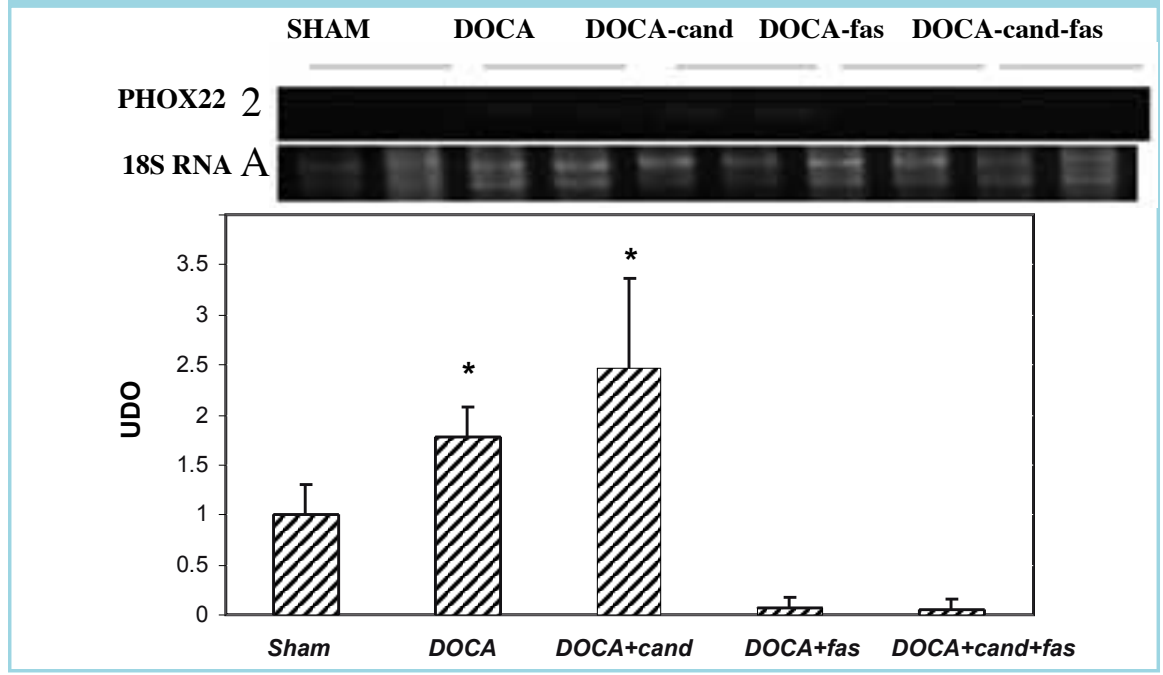

Figura 2: Expresado como unidades de densidad óptica (UDO). En panel superior se observan $m R$ NAs representativos de cada grupo. Promedio $\pm E S ; N=8-12$ / grupo. Símbolos: $*=p<0.05$ vs los grupos Sham, Doca + Fasusil y DOCA + Candesartán + Fasudil, post ANOVA significativo

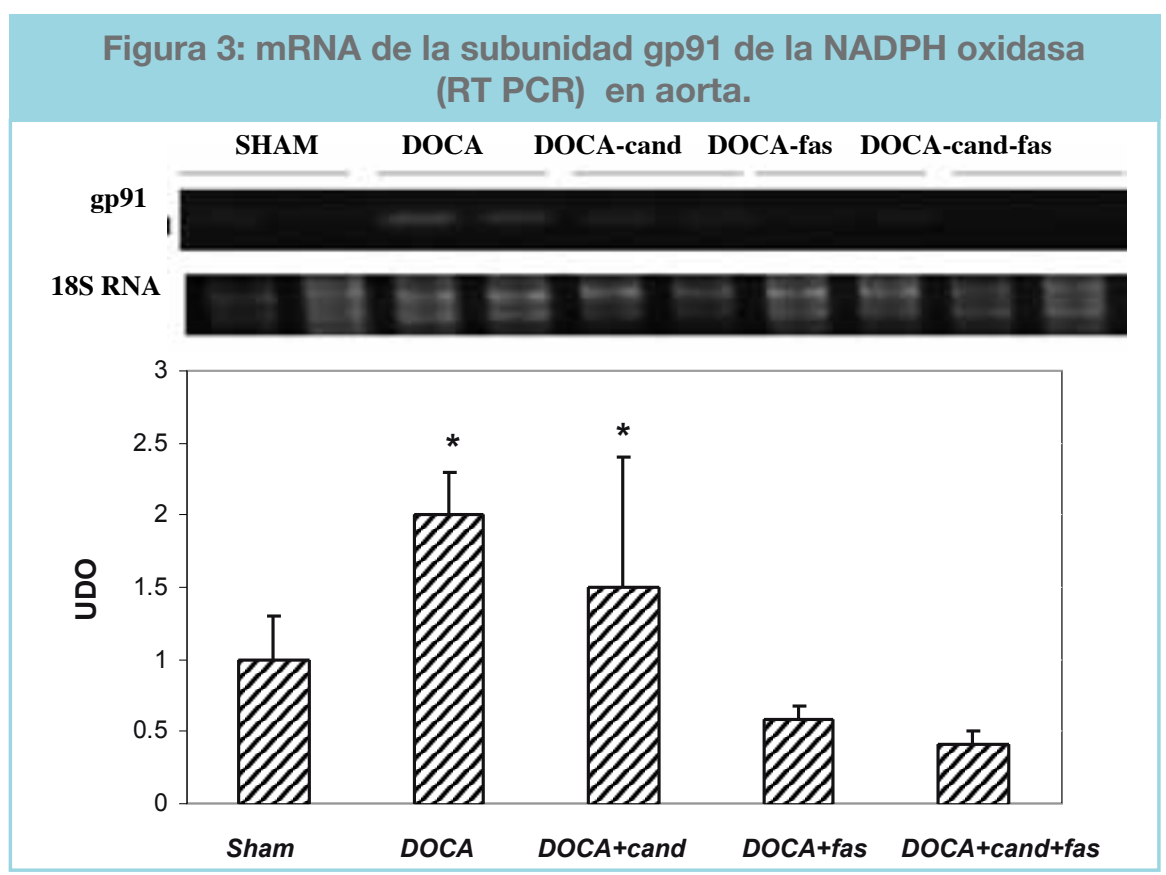

Figura 3: Expresado como unidades de densidad óptica (UDO). En panel superior se observan $m R$ $N A s$ representativos de cada grupo. Promedio $\pm E S ; N=8-12 /$ grupo. Símbolos: $*=p<0.05$ vs los grupos Sham, Doca + Fasusil y DOCA + Candesartán + Fasudil, post ANOVA significativo 
Niveles proteicos de TGF $\beta$ : Los niveles proteicos de TGF $\beta$ en la pared aórtica fueron 4 veces mayores en la ratas hipertensas DOCA con respecto de las ratas sham ( $p<0.05$, Figura 4). Después de tres semanas de tratamiento con Candesartán, Fasudil o con la combinación de ambos, los niveles proteicos de TGF $\beta$ fueron similares en los tres grupos DOCA con tratamiento respecto de las ratas Sham, sin diferencias entre ellos (Figura 4). Células inflamatorias EDl en anillos aórticos: El nú- mero de células inflamatorias ED1 en anillos aórticos fue en promedio $3.7 \pm 0.4$ células/anillo en las ratas controles normotensas (sham) y aumentó en las ratas DOCA en 6 veces ( $p<0.05$, Figuras $5 a$ y b). Después de tres semanas de tratamiento con Candesartán, Fasudil o con la combinación de ambos, el número de células ED1 en anillos aórticos fue significativamente menor que en las ratas DOCA sin tratamiento, pero mayor que en las ratas controles normotensas (Figura 5b).

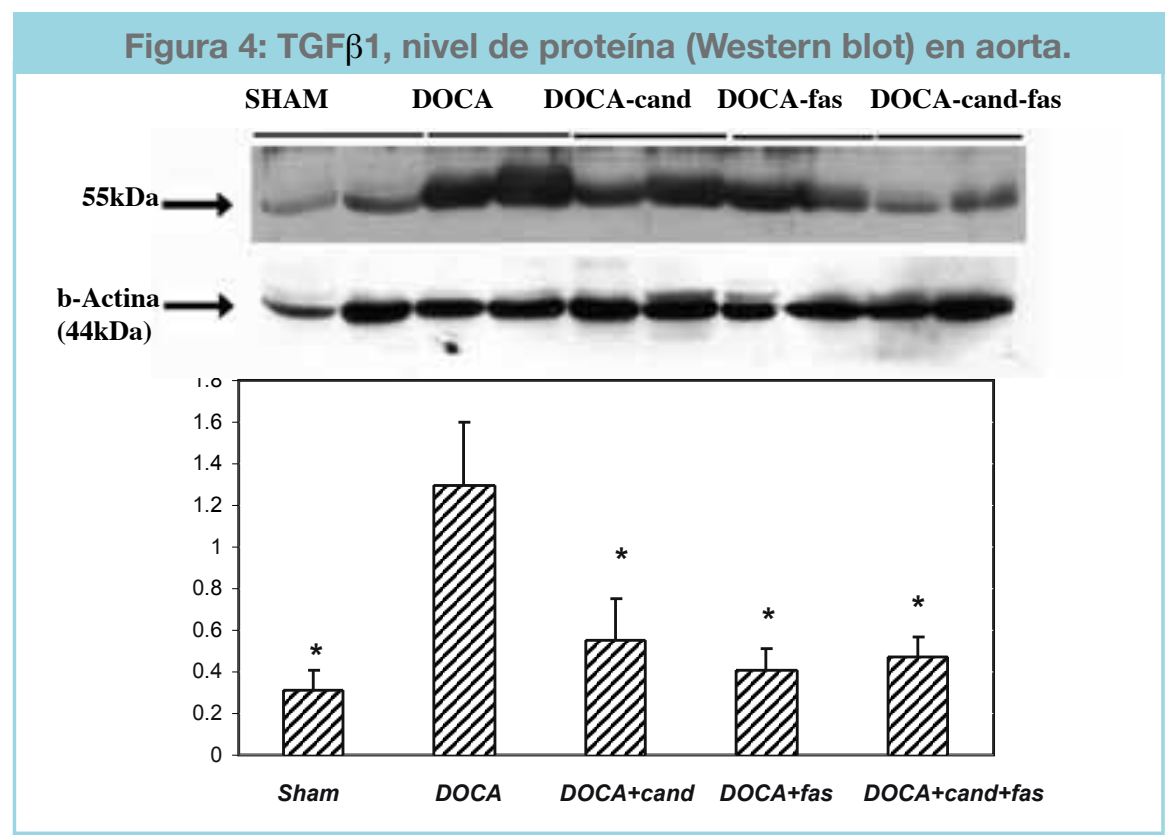

Figura 4: Datos normalizados por $\beta$ actina. En panel superior se observan Western blots representativos en cada grupo. Promedio $\pm E S ; N=8-12$ / grupo. Símbolos: $*=p<0.05$ vs grupo DOCA, post ANOVA significativo.

Figura 5a: Células inflamatorias ED1 en anillos aórticos (por inmunohistoquímica, con peroxidasa anti-peroxidasa y hematoxilina, Aumento 400X).



Figura 5a: Se muestra una sección de aorta representativa por cada grupo. $A=$ Sham, $B=D O C A, C$ $=$ DOCA + candesartán, $D=D O C A+$ fasudil, $E=D O C A+$ candesartán + fasudil . Las flechas negras señalan células ED1+ 


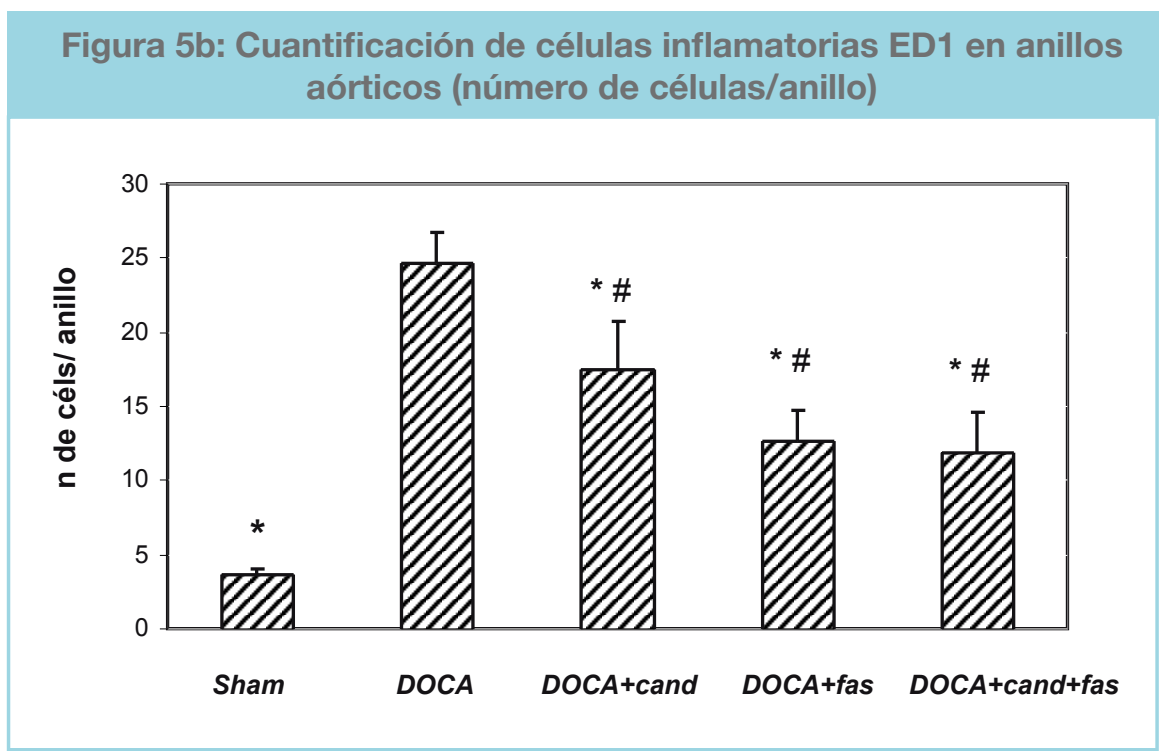

Figura 5b: Promedio $\pm E S ; N=8-12$ / grupo. Símbolos: $*=p<0.05$ vs DOCA, post ANOVA significativo, $\#=p<0.05$ vs Sham, post ANOVA significativo.

\section{Discusión:}

Los hallazgos más relevantes del estudio fueron: 1) En este modelo de HTA experimental hay aumento marcado de la actividad vascular aórtica de ROCK en paralelo con aumento de la expresión génica de NADPH oxidasa y de remodelado vascular patológico (aumento de células inflamatorias ED1 y de TGF $\beta$ ), 2) el tratamiento antihipertensivo disminuye la actividad aumentada de ROCK y previene/regresa en la pared vascular la inflamación y la sobrexpresión génica de TGF $\beta$ y 3) la sobrexpresión génica de NADPH oxidasa en la pared aórtica se normaliza sólo al inhibir en forma directa Rho kinasa con un inhibidor específico y no depende de un efecto antihipertensivo ni del bloqueo del receptor de AT1.

El aumento de la expresión génica de dos subunidades de NADPH oxidasa (p22 Phox y gp 91), que se ha observado en este trabajo, posiblemente producirá mayor cantidad de aniones superóxido y por lo tanto más stress oxidativo en la pared arterial. En este caso en particular esto podría deberse a mayor inflamación de la pared vascular inducida por la HTA y documentada con un mayor número de células ED1 en la pared arterial. La inflamación puede estimular stress oxidativo, la cascada de TGF- $\beta$ /colágeno y la producción de citokinas. En nuestras ratas hipertensas DOCA sin tratamiento también hemos observado aumento significativo de TGF- $\beta$ en la pared aórtica.
Para evaluar el efecto de fasudil sobre función endotelial en HTA e hipercolesterolemia experimental en ratas hipertensas por DOCA y dieta alta en colesterol Shah et al. (12), midieron la expresión génica de p22phox, niveles de ácido tiobarbitúrico y de anión superóxido en aorta. Fasudil y atorvastatina previnieron marcadamente la hipercolesterolemia y la alteración de la relajación endotelio dependiente, el daño endotelial, la disminución de la expresión génica de eNOS y de la relación nitritos/nitratos en suero, y el aumento del mRNA de p22phox, de anión superóxido, y sustancias reactivas de tiobarbitúrico sérico. El efecto protector de fasudil fue prevenido por L-NAME. Así, al inhibir ROCK mejora la disfunción endotelial debida a HTA e hipercolesterolemia ${ }^{12}$.

El tratamiento antihipertensivo con candesartán, fasudil o con ambos en forma combinada, normalizó la presión arterial, la actividad vascular de ROCK y los niveles de TGF $\beta$ en la pared arterial. También se observó reducción significativa de las células inflamatorias ED1 en la pared aórtica con candesartán, con fasudil y con ambos bloqueadores en forma combinada. Sin embargo, no ocurrió lo mismo con la expresión génica vascular de las 2 subunidades de NADPH oxidasa que se determinaron. En este caso, la sobrexpresión génica de las subunidades p22 Phox y gp 91 de la NADPH oxidasa en la pared aórtica observada en las ratas hipertensas sin tratamiento no se redujo al normalizar la presión arterial ni al bloquear el receptor de 
angiotensina 1 con candesartán exclusivamente. Esta sobreexpresión génica vascular de las subunidades p22 Phox y gp 91 de la NADPH oxidasa se normalizó solamente inhibiendo en forma directa Rho kinasa con un inhibidor específico y no depende de un efecto antihipertensivo ni del bloqueo del receptor de AT1), ya que la inhibición indirecta de ROCK al normalizar la PA con candesartán, no tuvo este efecto.

En ratas normotensas con niveles genéticamente elevados de enzima convertidora de angiotensina y de angiotensina II hemos observado activación de ROCK en la pared aórtica, mayor stress oxidativo y expresión génica de TGF $\beta$. También observamos en ese modelo experimental que el inhibidor de ROCK fasudil normaliza los niveles elevados de stress oxidativo vascular y la sobrexpresión de promotores de remodelado patológico (TGF $\beta$, PAI-1 y MCP1) ${ }^{9}$.

Nuestros datos parecen sugerir que desde el punto de vista del remodelado vascular patológico hipertensivo, la inhibición de ROCK podría tener efectos adicionales más eficientes que el tratamiento antihipertensivo a través del receptor de angiotensina 1. Esto es solamente una sugerencia interesante que debe ser evaluada con más estudios, en distintos modelos experimentales de HTA, frente a otros fármacos bloqueadores del sistema renina angiotensina, determinando un número mayor de parámetros de remodelado vascular (eventualmente también cardíaco y renal) y con un correlato funcional. Por otro lado, es posible que la inhibición de ROCK en la HTA tenga efectos protectores sobre el remodelado vascular más allá de la normotensión.

\section{Referencias :}

1. CHOBANIAN AV, BAKRIS GL, BLACK HR, CUSHMAN WC, GREEN LA, IZZO JL JR, el al. Joint National Committee on Prevention, Detection, Evaluation, and Treatment of High Blood Pressure. National Heart, Lung, and Blood Institute; National High Blood Pressure Education Program Coordinating Committee Seventh report of the Joint National Committee on Prevention, Detection, Evaluation, and Treatment of High Blood Pressure. Hypertension 2003; 42: 1206-1252.

2. MANCIA G, LAURENT S, AGABITI-ROSEI E, AMBROSIONI E, BURNIER M, CAULFIELD MJ, et al. Reappraisal of European guidelines on hypertension management: a European Society of Hypertension Task Force document. Journal of Hypertension 2009, 27: 2121-2158

3. ENS, Encuesta Nacional de Salud 2003, MINSAL. Disponible en: ttp://epi.minsal.cl/epi/html/invest/ENS/ ENS.htm

4. JALIL J, LAVANDERO S, CHIONG M, OCARANZA MP. Rho/Rho kinase signal transduction pathway in cardiovascular disease and cardiovascular remodeling. Rev Esp Cardiol. 2005; 58: 951-961.

5. SHIMOKAWA H, RASHID M. Development of Rhokinase inhibitors for cardiovascular medicine. Trends in Pharm Sciences 2007; 28: 296-302
6. SHIMOKAWA H, TAKESHITA A. Rho-kinase is an important therapeutic target in cardiovascular medicine. Arterioscler Thromb Vasc Biol. 2005; 25: 1767-1775.

7. LOIRAND G, GUERIN P, PACAUD P. Rho kinases in cardiovascular physiology and pathophysiology. Circ Res. 2006; 98: 322-334.

8. CALO LA, PESSINA AC. RhoA/Rho-kinase pathway: much more than just a modulation of vascular tone. Evidence from studies in humans. J Hypertens. 2007; 25: 259-264.

9. RIVERA P, OCARANZA P PAZ, LAVANDERO S, JALIL JE. RHO Kinase Activation and Gene Expression Related to Vascular Remodeling in Normotensive Rats With High Angiotensin-I-Converting Enzyme Levels. Hypertension. 2007; 50: 792-798

10.- KUSAKA I, KUSAKA G, ZHOU C, ISHIKAWA M, NANDA A, GRANGER DN, et al. Role of AT1 receptors and $\mathrm{NAD}(\mathrm{P}) \mathrm{H}$ oxidase in diabetes-aggravated ischemic brain injury. Am J Physiol Heart Circ Physiol. 2004; 286: H2442-H2451.

11.- STERNBERGER LA, STERNBERGER LH. The unlabeled antibody method: comparison of peroxidaseantiperoxidase with avidin-biotin complex by a new method of quantification. J. Histochem. Cytochem. 1986; 34: 599-605.

12. SHAH DI, SINGH M. Involvement of Rho-kinase in experimental vascular endothelial dysfunction. Mol Cell Biochem. 2006; 283: 191-199. 